\title{
Long term evaluation of wetting-drying cycles for compacted soils treated with lime
}

\author{
Maafi Nabil ${ }^{1}$, Akchiche Mustapha ${ }^{1}$, Sara Rios ${ }^{2}$ \\ ${ }^{I}$ Department of Civil Engineering, LEEGO, University of Houari Boumedien (USTHB), Algiers, Algeria. \\ maafinabil@ymail.com \\ ${ }^{2}$ CONSTRUCT-GEO, Faculdade de Engenharia (FEUP), Universidade do Porto, Portugal.
}

\begin{abstract}
This article addresses the results of experimental study behavior of recycled soil treated with lime and exposed to wettingdrying cycles at the long term. The object of this research is to focus on effect of a wide range of lime content on evolution of volume and durability of stabilized soils, when exposed to wetting- drying cycles. In this work, an experimental study was performed on soils which were treated with 8 percentages (0-8\%) of lime. Treated soils were conserved for three different periods of cure: 7, 28 and 180 days, and then exposed to 12 wetting-drying cycles of 48 hours for each cycle. However, the behavior under wetting-drying cycles was only satisfactory for lime percentages above $6 \%$. The formation of C-S-H and C-A$\mathrm{H}$ responsible for the increased strength of stabilized soil samples are showed and the expensive ettringite was also responsible for the decreased strength of stabilized soil samples.
\end{abstract}

Key words: soil, treatment, lime, long term.

\section{Introduction}

Soil treatment techniques have been known for more than 60 years in the field of earthworks, in the construction of embankments and for roads (highways, railways, road, airports, etc.). The treatment method by lime is an ecological and sustainable method, currently it is considered as an important method for reuse and valuation of unusable soil. Exposure to repetitive environmental conditions present one of the most destructive actions that may damage infrastructures, because their mechanical properties are severely affected and the consequence is; crack propagation and instability, are occurred in geotechnical works, the most destructive actions are freezing-thawing [1] and wetting- drying cycles [2,3]. However, results of some researches on the effects of drying-wetting cycles on the stability and durability of soils are very disparate and depend on the type of soil, the percentage of stabilizer, test methods and cure conditions [4]. Treatment with lime has double effects, the first is to improve soil properties (in short term), and the second is to stabilize the soil (in the medium and long term) [5]. The immediate effect of the addition of lime causes the flocculation and the agglomeration of clay particles caused by cation exchange on the surface of soil particles. The result of this reaction in short term is mainly to improve workability and plasticity of soil wish improves its handling and its implementation by compaction to achieve the required capacity of soils $[6,7]$. In the long term; reactions may require time from a week to months or even a year to complete, depending on the speed of chemical decomposition and hydration of aluminates and silicates. This process results in the formation of cementitious materials which bind the soil particles and improve the mechanical properties of treated soil. Stabilization is a process of change (usually in the medium or long term), the characteristics of soil to bring it to final state of stability, particularly against water action, and resulted in a strong soil. It retains its original form, its stability and its quality when it exposed to the environment and maintains adequate residual strength at long term and provides sufficient strength to climate condition. The lack of knowledge about stabilization due to the reaction between lime and soil, and about behavior of soils treated with lime at in the long-term, and subject to cycles of drying-wetting are the reasons behind the lack of the use of this technique. In-depth research aiming at acquiring more knowledge should allow expanding the scope of the lime treatment in earthworks for the embankment bases in wet and floodplain zones.

\section{Materials and testing methods}

Support materials of this study are; natural soils classified according to ASTM unified classification system D 2487 (ASTM, 2000) as indicated in Table 1. The soil samples were collected from north Algeria of Sidi Aissa region of M'Sila (SAM). The untreated soil X-ray diffractogram is shown in Figure 1. The soil mainly includes illite, kaolinite, chlorite, Smectite and quartz minerals. Tables 1 and 2 present the physical and chemical properties of soils. Lime used for the treatment of the different soils is quicklime $(\mathrm{CaO})$, delivered by the ERCO company located in SAIDA, ALEGIRIAS. The physical and chemical characteristics of lime are given in the Table 3. 


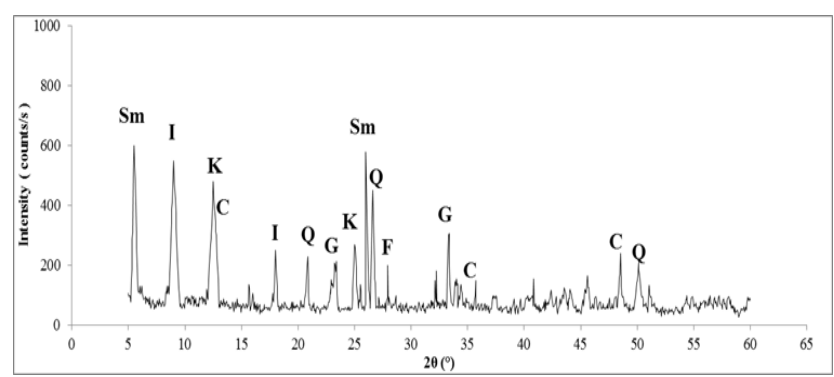

Figure 1. XRD patterns of the soil.

Table1. Geotechnical properties of the studied soils.

\begin{tabular}{cc}
\hline Soils index & SAM \\
\hline Parameters & $\begin{array}{c}\text { Mean } \\
\text { values }\end{array}$ \\
Liquid limit (ASTM D 4318 00) & 61 \\
& \\
Plastic limit (ASTM D 4318 00) & 30 \\
Plasticity index (ASTM D 4318 00) & 31 \\
Methylene blue value (ASTM C837) & 6.2 \\
Grain size (\%) > 0.075mm & 84 \\
Clay content (\%) (ASTM D 422 61) & 22 \\
pH (ASTM D6276 - 99A) & 8.46 \\
& \\
Free swelling (ASTM D 4546) (\%) & 4 \\
(UCSC) ASTM D2487 & CH \\
\hline
\end{tabular}

Table2. Chemical composition of the studied soils.

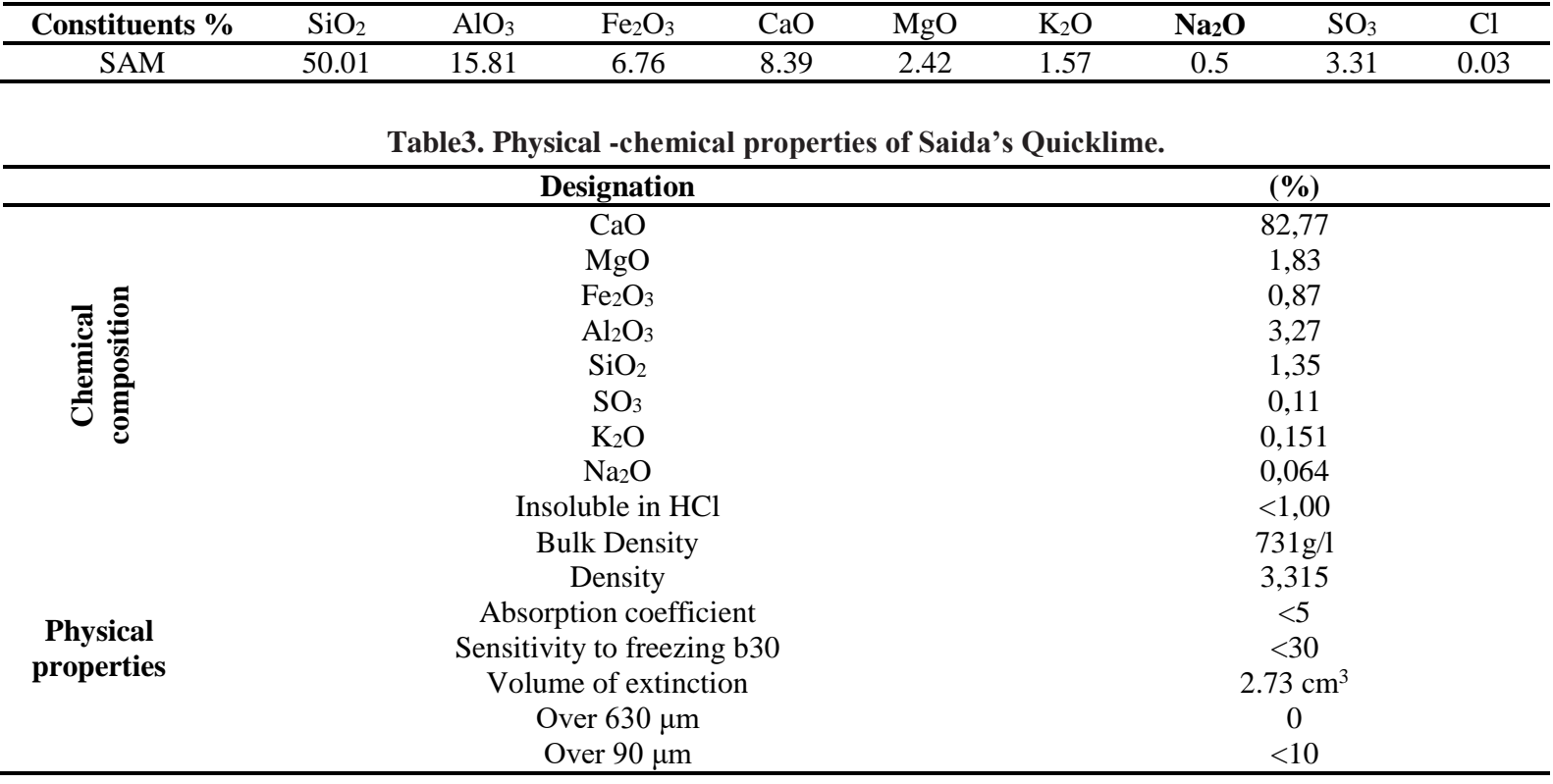

\section{Experimental Results}

The variation in volume of studied soils at different periods of cure such as 7, 28 and 180 days exposed to dryingwetting cycles are presented in Figure 2. The increase in number of solicitation drying-wetting, affect significantly the volume of treated and untreated soils. After 7 days of curing; there was a decrease of volume for all soils treated and untreated with lime and a different level of volume change was observed. The untreated soil presents an important damage during the $1^{\text {st }}$ cycle of drying-wetting. The sample with 1 and $2 \%$ of lime are deteriorated during the $1^{\text {st }}$ cycle and the sample with 3 and $4 \%$ of lime, we not an important damage during the $\left(2^{\text {nd }}\right.$ and $\left.3^{\text {rd }}\right),\left(4^{\text {th }}\right.$ and $\left.5^{\text {th }}\right)$ and $\left(7^{\text {th }}\right.$ and $\left.8^{\text {th }}\right)$ cycle after 7,28 and 180 days respectively, and for the percentage above $4 \%$, they show 
satisfying behavior to the last cycle. A decrease about 78.62, 75.62 and $66.32 \%$ in volume is noted with $3 \%$ of lime after 7, 28 and 180 days respectively. However, soils with $8 \%$ lime show a good relation between dryingwetting cycles and a loss in volume; 22.27, 13.22 and $9.23 \%$ at 7,28 and 180 days respectively.
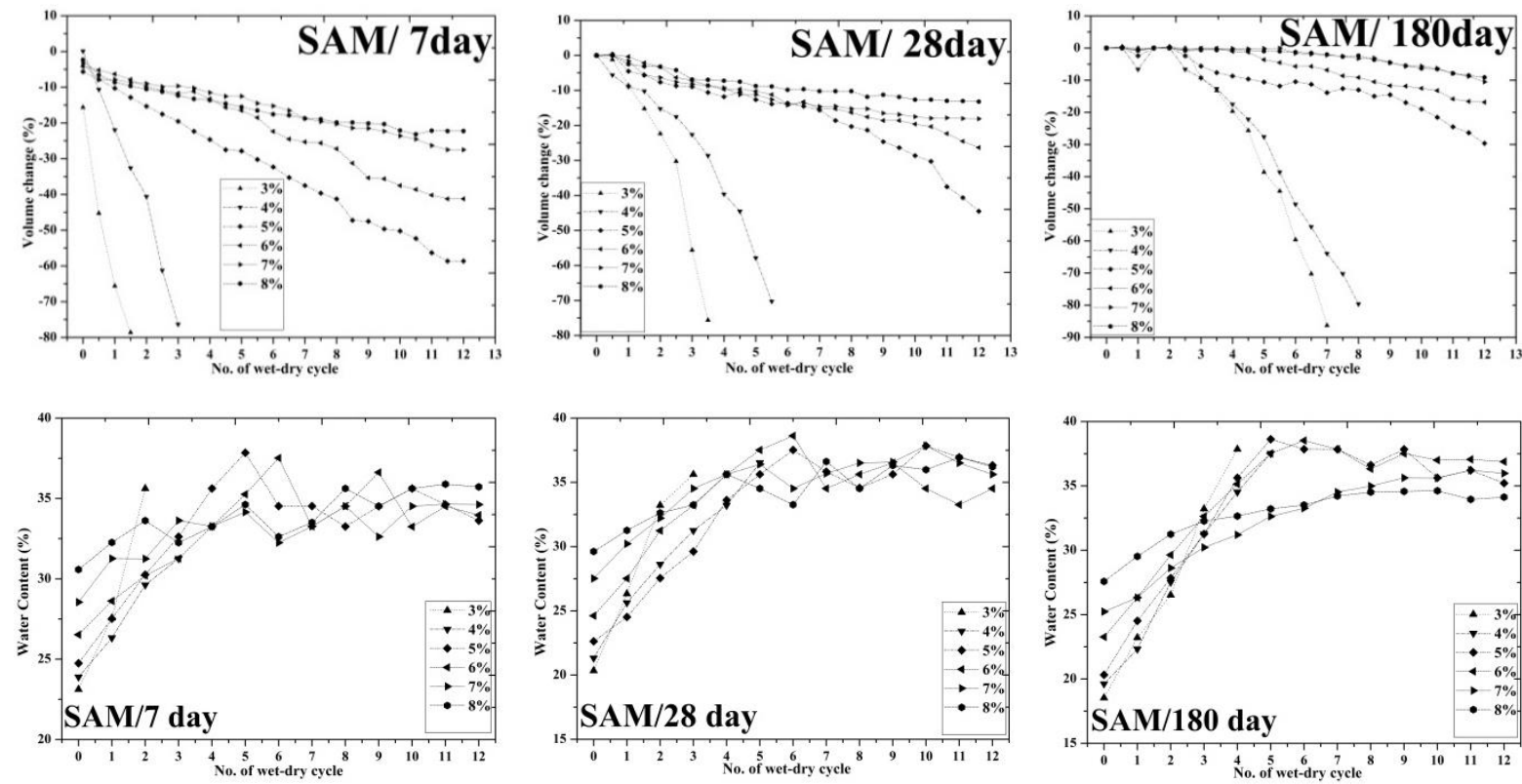

Figure 2. The loss of volume and water content changes with wetting and drying cycles of studied soils.

After 7 days of cure, the variation in water content is clear, and water content increases after each cycle for studied soils with low lime contents (3 and 4\%). Lime contents higher than $4 \%$, water content rests almost stable after $7^{\text {th }}$ cycle for soil. On the other hand, it can be observed that the water content decreases when the lime content increases. The effect of lime addition is especially visible in soils cured to 28 and 180 days, where the water content rests almost stable for all lime percentages in soil. The soil is a porous material, so it has the ability to absorb water by capillarity, and to maintain this water during the humidification. After drying cycle, they showed a crack due to shrinkage and low tensile strength. When the water content decreases due to the repetitive exposition to drying cycle, suction forces increase until the tensile stress is equal to the cohesive forces. At this stage, the formation of cracks allows more shrinkage during visual observation of the tested samples, with a loss of additional water; primary cracks grow and let formation of new cracks. The external surface of soil samples will also be affected due to the rapidly loss of water at the surface and inside of this the samples. At the beginning of the second wetting cycle, cracks are not closed or partially closed on the surface, so that cracking are not completely reversible process (rebounding) during wetting-drying cycles, new cracks can therefore be created in soils so that during the next drying cycle, cracks occurs probably in the same spot. Crack propagation causes a loss of strength and subsequently accelerates the degradation of samples with water absorption. This degradation differs according to rate of lime used during the treatment of these soils. Treated soils showed low propagation of fissures due to the formation of new hydrated C-S-H which limiting and reducing the development of cracks, when they are subjected to the drying-wetting cycle compared to untreated soils. The treatment with lime of studied soils has the effect to improve the resistance when these soils were exposed to the drying-humidifying cycle. This effect is very remarkable especially with import periods of cure (superior than 28 days), as a consequence allowing the development of pozzoulanic reactions and formation of new hydrates C-S- H, that participate to reduction of porosity and consequently, the reduction of the water absorption capacity of soils treated with lime, so that at the end we obtain stable soils were obtained as a result.

The XRD analyzes of the lime-treated soil for 180 days at $20^{\circ} \mathrm{C}$ and exposed to a wetting-drying cycle are illustrated in Figure 3. The pozzolanic reactions are the results of the combination between the silica and the alumina of the soil with lime calcium to the presence of water. As long as the residual calcium is available and the $\mathrm{pH}$ is high enough, the pozzolanic reaction will continue. These reactions result in the formation of calcium hydrates due to the presence of $\mathrm{Ca}^{+2}, \mathrm{OH}^{-}$and $\mathrm{SiO}_{4}$ and $\mathrm{AlO}_{6}$ ions [1]. Identification revealed the formation of 
new calcium hydrates (CSH and $\mathrm{CAH}$ ) in all the lime-treated samples and ettringite in samples with low lime content.
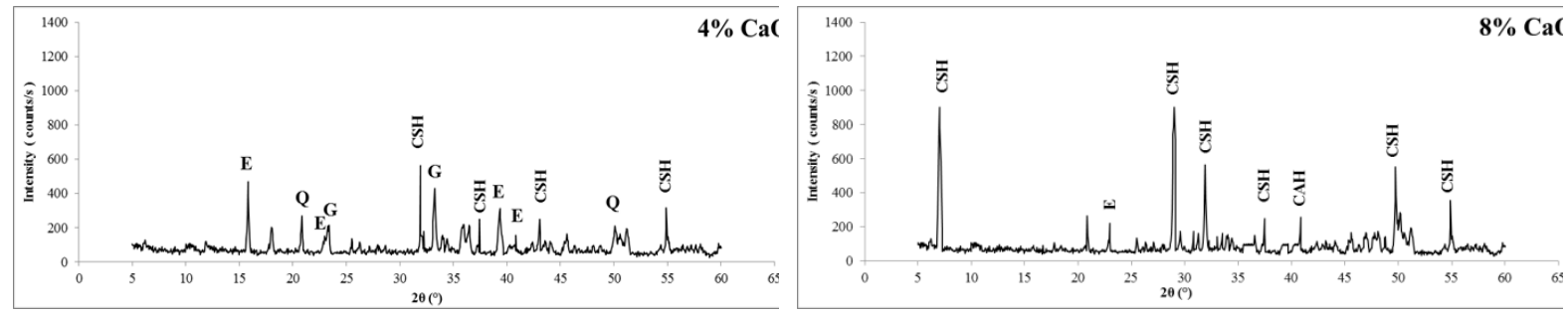

Figure 3. XRD patterns of the soil after 180 days of curing at the end of wetting-drying cycles Exposure to repetitive wetting-drying cycles led to an increase in the macropores (pore diameter more than $6 \mu \mathrm{m}$ ) are illustrated in Figure 4 due to additional ettringite formation for soil samples with $4 \%$ of lime. A slight reduction in the volume of micropores (pore diameter less than $0.3 \mu \mathrm{m}$ ) for soil samples with $8 \%$ of lime can also be observed.
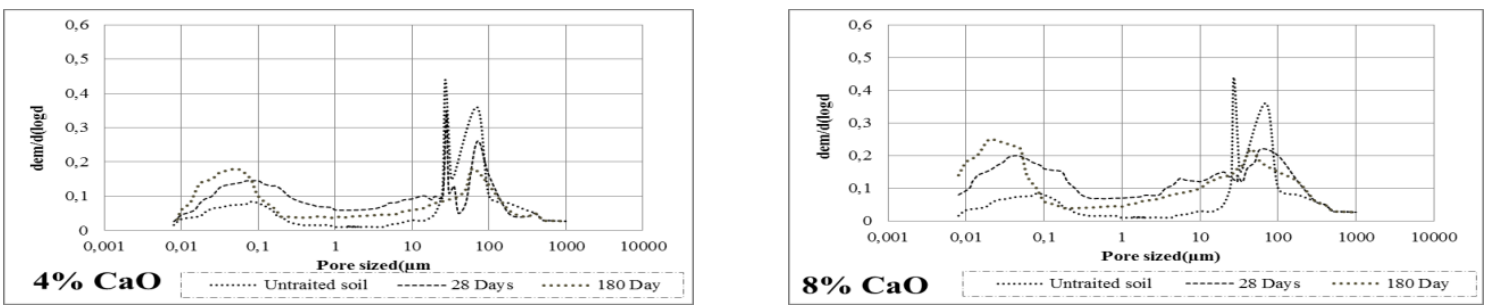

Figure 4. Pore size distribution of the soil samples at the end of wetting-drying cycles.

\section{Conclusions}

The cyclical exposure drying-wetting of investigated soils allows following two determining indicators of the durability (volume change and the rate of water absorbed) depending on the rate of lime and period cure of studied soil.

Measuring the rate of water absorption collected soils at the time of the cyclic solicitations makes it possible to observe a disproportional relationship between the rate of water absorbed and lime. For superior percentage of lime; treated soils showing low water absorption rate confirm their low volume variation. The same behavior was observed for all soils with different cure periods. For inferior percentage of lime, the studied soils indicate an opposite behavior, from where their large volume variation.

The two distinguishing behaviors are due to the low permeability, caused by the decrease of the porosity due to the cementation following the pozzolanic reaction between mineral of sol and lime by the formation of new hydrated C-S-H and C-A-H responsible of decrease the pores and propagation of cracks.

\section{References}

[1] Aldaood M. Bouasker M. AL-MUKHTARET 2014. Impact of wetting-drying cycles on the microstructure and mechanical properties of lime-stabilized gypsums soils, Engineering Geology 174 (2014) 11-21.

[2] Al-Obaydi, M.A., Al-Kiki, I.M., Al-Zoubaydi, A.H., 2010. Strength and durability of gypsums soil treated with waste lime and cement. J. Al-Rafidain Eng. 18 (1), 28-42.

[3] N. Maafi, M. Akchiche, R. Sara. "Chapter 5 Wetting and Drying Compacted Soil-Lime Mixtures", Springer Nature,

[4] Tang, A.M., Ngoc, M., Cui, Y.J., 2011. Effects of the maximum soil aggregates size and cyclic wetting-drying on the stiffness of a lime-treated clayey soil. J. Geotech. 61 (5), 421-429.

[5] Lemaire, K., Deneele, D., Bonnet, S., Legret, M., 2013. Effects of lime and cement treatment on the physicochemical, microstructural and mechanical characteristics of a plastic silt. Eng. Geol. 166 (8), 255-261

[6] Alrubaye, A. J., Hasan, M., Fattah, M. Y., (2016), "Improving Geotechnical Characteristics of Kaolin Soil Using Silica Fume and Lime", Special Topics \& Reviews in Porous Media- An International Journal, Vol. 7 (1), pp. 77-85.

[7] Alrubaye, A. J., Hasan, M., Fattah, M. Y., (2017), "Stabilization of Soft Kaolin Clay with Silica Fume and Lime", International Journal of Geotechnical Engineering, Vol. 11, No. 1, pp. 90-96, Taylor \& Francis. 